\title{
OPTIMIZATION OF QUATROPOD JACKET SUPPORT STRUCTURES FOR OFFSHORE WIND TURBINES SUBJECT TO SEISMIC LOADS USING GENETIC ALGORITHMS
}

\author{
Mohammad H. AlHamaydeh ${ }^{1}$, Samer A. Barakat ${ }^{2}$, and Omar M. Nassif ${ }^{2}$ \\ ${ }^{1}$ Department of Civil Engineering \\ American University of Sharjah \\ P.O. Box 26666, Sharjah \\ United Arab Emirates \\ e-mail: malhamaydeh@aus.edu \\ ${ }^{2}$ Department of Civil Engineering \\ University of Sharjah \\ P.O. Box 27272, Sharjah \\ United Arab Emirates \\ \{sbarakat,onaseef\}@sharjah.ac.ae
}

Keywords: Offshore Support Structures, Genetic Algorithms, Optimization.

\begin{abstract}
This paper presents evolutionary-based least-weight topology and size optimization procedure for designing offshore wind turbine supporting structures. A modified version of Genetic Algorithms is presented herein. The GA technique is used for solving the nonlinear constrained optimization problem of $3 D$ quatropod jacket offshore support structure. In this optimum design formulation, the objective function is the material weight of the supporting truss; the design variables are the locations of the ends of the truss members as well as the cross-sectional areas of the truss members. The constraints are the stresses in members and the displacements of the joints. The constraints are handled using non-stationary (dynamically modified) penalty functions. The quatropod jacket, one of the most commonly used design alternatives for offshore supporting structures is presented as demonstration to the efficiency of the presented GA algorithm. The quatropod jacket test problem is subjected to gravity, wind, wave and earthquake loading conditions. The results show that the GA method is efficient in finding the best discovered optimal solution [structural topology and weight].
\end{abstract}




\section{INTRODUCTION AND BACKGROUND}

Often times, the individual design solutions (typically, a range of possible feasible design solutions exist for a given engineering problem) significantly influence the structural response to the environmental loads and thus impose additional constraints to the design. For example, the type, configuration, and structural member sizes of the supporting offshore structure, could potentially have a dramatic impact on marine growth size which greatly impacts the hydrodynamic drag forces. As a result, the nonlinearities associated with fluid-structure interaction may be substantial and no longer be admissible to dismiss during the design stage [1].

In the presence of multiple optima and non-smooth constraints in the design variable space, it is difficult to obtain a set of optimum values using member-level optimization formulations. This shortcoming inspired researchers to explore using the relatively new and innovative evolutionary-based optimization techniques. For example, the following methods have been used recently to address structural engineering optimization problems: Shuffled Complex Evolution Optimizer (SCEO), Ant Colony Optimization (ACO), the Genetic Algorithms (GA), and Particle Swarm Optimizer (PSO) (e.g. [2]-[12]).

Many structural optimization problems involve problem-specific constraints applicable to the solutions limiting the feasible search space. In these types of problems, it is challenging to adapt traditional optimization techniques to handle constraints. One of the most popular constraint handling methods is through penalty functions due to the relative simplicity and ease of implementation. Topology optimization was used by many researchers to generate alternative structural design concepts for benchmark wind turbine blades. In these studies, the focus is alternative structural layout for wind turbine blades with the aim of improving its design, minimizing weight and ultimately, wind energy cost reduction (e.g. [13]-[23]).

This study presents an efficient Genetic Algorithm with Domain Trimming (GADT) optimization technique to achieve superior optimization. The GADT scheme is used to solve a typical least-weight design for offshore wind turbine supporting structures through topology and member size optimization. The algorithm is coded using the MATLAB commercial software package [24] and its built-in GA libraries; while the structural analysis and design for the different topology and loading configurations is performed using the SAP2000 commercial software package [25]. The linkage and automation of the structural analysis and design tasks are initiated from within the MATLAB code and established through the Open Application Programming Interface (OAPI) capabilities of the SAP2000 software.

\section{GENETIC ALGORITHM WITH DOMAIN TRIMMING (GADT)}

To begin GA optimization, a population of $\mathrm{N}_{\mathrm{p}}$ (population size) solution alternatives are generated randomly using a uniform probability distribution; each solution of the GA consists of a combination of variables $\left(\mathrm{x}_{1}, \mathrm{x}_{2}, \mathrm{x}_{3}, \ldots, \mathrm{x}_{\mathrm{n}}\right)$ which has its own fitness value. In cases where the optimization is performed to find the minimum of a given problem, lower fitness is preferable. Solutions that yield low objective values would have better fitness as long as they are not penalized for violating the constraints which would result in increasing the fitness value. Populations of solutions are represented by chromosomes or variable strings. In this research, a variable string is used instead of a binary string because the design variables are discrete. Once the fitness for every solution in the current population is calculated using the fitness function $\boldsymbol{F}_{\boldsymbol{j}}(\boldsymbol{X})$, unfit solutions are eliminated. Any solution whose fitness is greater than the average, $\boldsymbol{F}_{\text {ave }}$, of the population is reassigned a fitness of zero: 
$F_{j}(X)=\left\{\begin{array}{ll}\mathrm{F}_{\text {ave }}-F_{j}(X) & \text { for } F_{j}(X)<\mathrm{F}_{\text {ave }} \\ 0 & \text { for } F_{j}(X) \geq \mathrm{F}_{\text {ave }}\end{array} \quad \mathrm{j}=1,2, \ldots, \mathrm{N}_{\mathrm{p}}\right.$

where $(\boldsymbol{X})$ is the vector of the design variables.

The three basic operations of a GA, reproduction, crossover, and mutation, are used to improve the fitness of each population from one generation (iteration) to the next (

Figure ). The reproduction operation selects the better fit designs, copies them and places them into a mating pool allowing each to mate and reproduce. Different selection methods are available in the literature. The roulette wheel selection method is used in this study for its simplicity and popularity. The uniform crossover operation is used to combine genetic information between two parent solutions. Uniform crossover selects two parent solutions at a time from the mating pool and swaps variables corresponding to zeros in a binary string known as a mask.
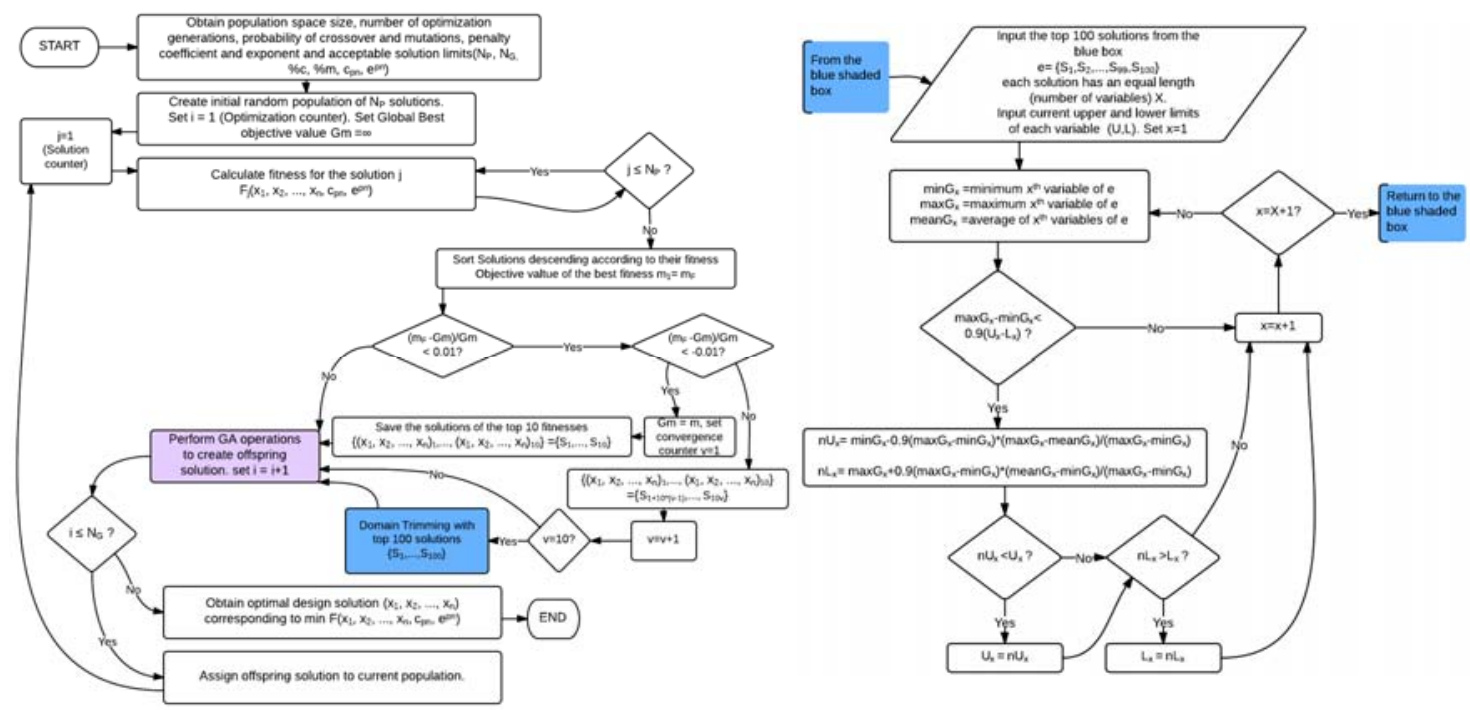

Figure 1: Flow chart of general GADT optimization [26]

A major shortcoming of GA optimization is that while it is good in global search of the optimum solutions, it is weak in local search; meaning that it will converge to the optimum solution but will not locate it with high precision especially if the population size is small. The reason is that initially, the solution variables are selected randomly from a pre-specified range (domain), those variables do not change but relocate from one solution to another, with the exception when mutation occurs which introduces new variables from the specified domain. However, the probability of mutation is generally kept low $(2 \%-5 \%)$ in order to increase the solution stability. Another shortcoming of the GA approach is the choice of domain; a poor choice of the domain (e.g.: $[1,1000]$ when the optimum value is 2 ) dramatically impacts convergence. In this study, a new technique that addresses these GA shortcomings is presented. The technique is to trim the domain so that the chance of selecting the optimal variable is enhanced (e.g.: trimming a domain from $[1,1000]$ to $[1,10]$ would increase the probability of selection of the optimal variable by 200 times). After trimming, the GA is reinitiated and continues to do so until the optimum solution is found. Figure 1 shows the flowchart for the GADT Method. For more details about GADT algorithm the reader is referred to [26]. 


\subsection{Constraints handling}

One of the most common constraint-handling techniques is through penalty functions. In such approaches, the problem is solved as an unconstrained one, where the constraints are penalized (such that non-feasible solutions are characterized by high function values). This allows for a single objective function to be formulated and in turn minimized using an unconstrained optimization algorithm. The penalty functions can either be stationary or nonstationary (dynamically modified). It is observed that non-stationary penalty functions are almost always superior to stationary penalty functions. A non-stationary penalty function is, generally, defined as

$$
f(X)=F(X)+p\left(X, c_{p n}, e_{p n}\right)
$$

Where $F(x)$ is the original objective function of the constrained optimization problem; $p(\bullet)$ is a dynamically modified penalty value, defined as

$$
p\left(X, c_{p n}, e_{p n}\right)=\left\{\begin{array}{r}
\left(c_{p n} r_{i}\right)^{e_{p n}}, \quad r \geq 1 \\
0, r<1
\end{array}\right.
$$

Where, $\boldsymbol{r}_{\boldsymbol{i}}$ is the individual member's demand over capacity ratio according to the design code. $\boldsymbol{c}_{\boldsymbol{p} \boldsymbol{n}}$ and $\boldsymbol{e}_{\boldsymbol{p}}$ are the penalty coefficient and exponent, respectively. As their name implies, they provide means to penalize the optimization objective if the ratio $\boldsymbol{r}$ exceeded unity. $\boldsymbol{e}_{\boldsymbol{p} n}$ serves as efficient means to greatly increase the penalty as $\boldsymbol{r}$ gets farther from unity while $\boldsymbol{c}_{\boldsymbol{p} n}$ ensures that values of $\boldsymbol{r}$ get farther away from unity. In this study, the empirically-selected values of the penalty coefficient and exponent are 2.5 and 2 , respectively.

\section{PROBLEM FORMULATION FOR OFFSHORE JACKET STRUCTURE}

The proposed optimization problem for the wind turbine supporting truss structure is quite complex as it aims at discovering the optimum geometrical configuration and shape as well as member sizes. The mathematical form of the problem can be expressed as follows:

$$
\begin{array}{ll}
\text { Find } & A^{T}=\left\{A, A_{2}, \ldots A_{h}, x_{s}, y_{s}, z_{s}\right\} \\
\text { To Minimize } & F=W\left(A, x_{s}, y_{s}, z_{s}\right)=\rho \sum_{i=1}^{n} L_{i} A \\
\text { Subject to } & g_{j}^{L} \leq g_{j}(A) \leq g_{j}^{U} \quad j=1,2, \ldots m \\
\quad \text { and } & A_{i}^{\min } \leq A_{i} \leq A_{i}^{\max } \quad i=1,2, \ldots n
\end{array}
$$

Where $\mathrm{Ai}=$ the design variable $\mathrm{i}$ (member i cross-sectional area), (xs, ys, zs) are the members' end nodes coordinates, $\mathrm{n}=$ the number of the design variables, $\mathrm{W}(\mathrm{A})=$ the objective function (the structural weight), $\rho=$ the material density, $\mathrm{Li}=$ the member length, $\mathrm{m}=$ the number of inequality constraints $(\mathrm{g}), A_{i}^{\min }$ and $A_{i}^{\max }$ are the lower and the upper bound of the $i^{\text {th }}$ variable respectively. The lower and upper bounds posed by Eq.(4) on the constraints include truss member stresses and joint displacements according to the specified design code.

Combining the objective and penalty functions together would yield the fitness function used in GADT:

$$
\operatorname{Min} F\left(A_{i}, x_{i}, y_{i}, z_{i}, c_{p n}, e_{p n}\right)=f\left(A_{i}, x_{i}, y_{i}, z_{i}\right)+p\left(A_{i}, x_{i}, y_{i}, z_{i}, c_{p n}, e_{p n}\right)
$$


For practicality and to constraint the structure's space, the locations of the nodes in the x-y plane are, not allowed to change. Instead, the vertical location $\mathrm{z}$ of the node along the legs is allowed to change (see Figure 2b). Consequently, the slope of the legs, the length of members, and the weight of the structure are changing as well.

\subsection{Quatropod Jacket}

One of the most commonly used offshore wind turbine support systems is used herein to demonstrate the efficiency of the GADT algorithm. The developed code is applied to design a 3D quatropod jacket trusses subjected to various loading conditions. A total lumped mass of $3.4 \times 10^{5} \mathrm{~kg}$ representing the turbine mass is assumed on the top of the tower. The design constraints include truss joint displacements and member stresses. The supporting truss systems are analyzed for load combinations and designed for conditions specified according to the 2010 AISC-LRFD design code [27].

\section{RESULTS AND DISCUSSION}

The GADT algorithm is applied to the quatropod structure. The effect of the GADT control parameters (population size, penalty coefficient and exponent) on the optimum solutions is explored and the combination producing the best results are adopted. This 64-bar 3D truss structure is doubly-symmetric about the $\mathrm{x}$ - and y-axes. Thus, the truss members are linked in 14 member groups. Figure 2 shows the detailed description of these groups. Therefore, the optimization problem has 33 independent design variables and 32 (13 tension stresses, 13 compression stresses, and 6 displacements) constraints. Table 1 gives the best discovered solutions for the structure.
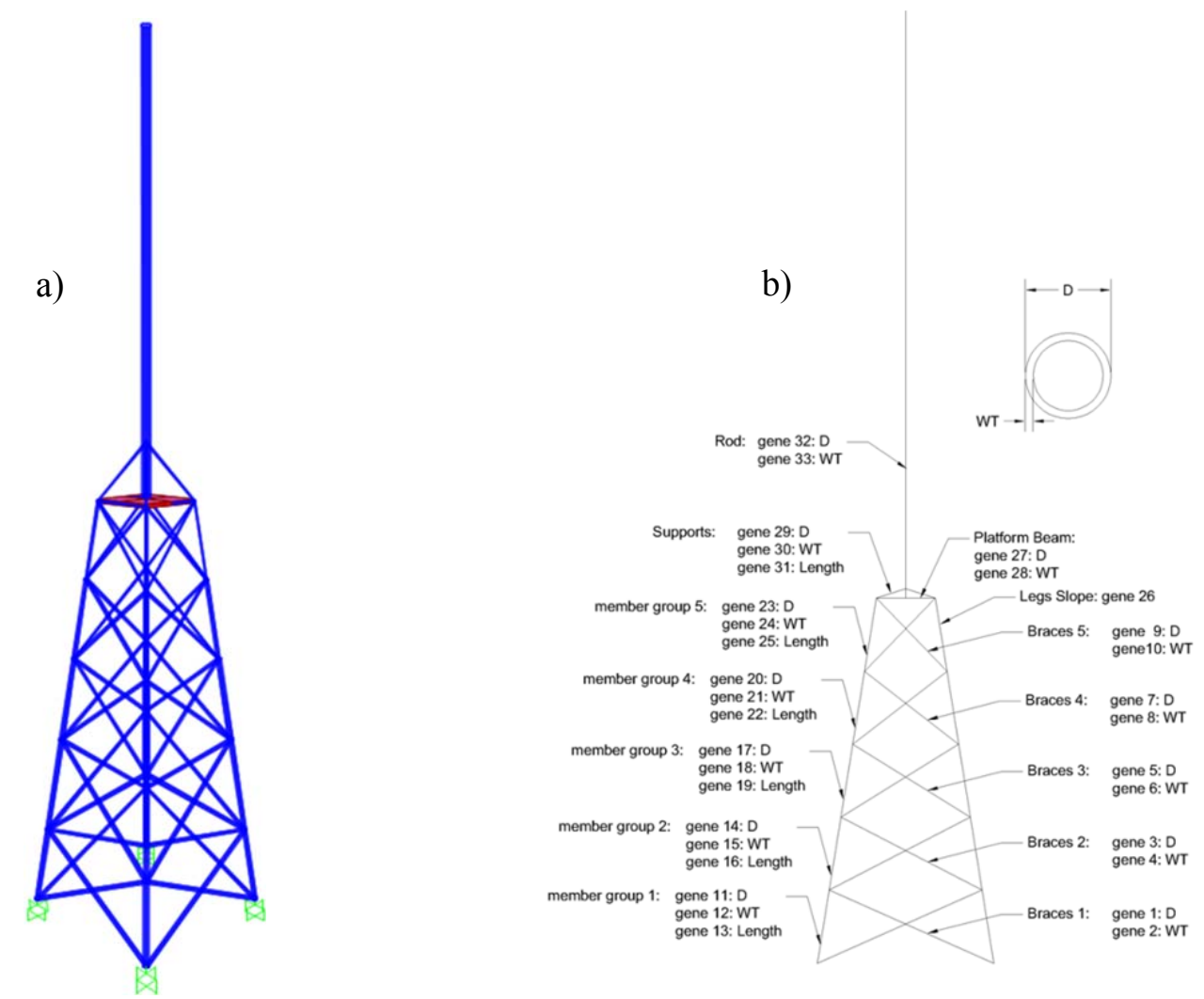

Figure 2: Wind turbine supporting truss structures: a) 3D View b) Description of the design groups (variables). 
The best discovered optimum weight via GADT algorithm is found to be 4,227,300 kgf. The best solution is obtained after approximately 500 searches shown in Figure 3. The optimal solutions found by the GADT meet all constraints and have no active constraints.

Table 1: Optimal Design values for the 3D 64-bar space truss (quatropod jacket).

\begin{tabular}{|c|c|c|c|c|c|c|c|c|}
\hline \multicolumn{9}{|c|}{ Optimal Design Variables (m) } \\
\hline \multicolumn{2}{|c|}{ Variable } & \multirow{2}{*}{$\begin{array}{c}\text { BDV } \\
0.74\end{array}$} & \multicolumn{2}{|c|}{ Variable } & \multirow{2}{*}{$\begin{array}{r}\text { BDV } \\
0.14 \\
\end{array}$} & \multicolumn{2}{|c|}{ Variable } & \multirow{2}{*}{$\begin{array}{r}\text { BDV }^{5} \\
0.57\end{array}$} \\
\hline 1 & $\mathrm{D}^{1}$ & & 12 & $\mathrm{WT}^{2}$ & & 23 & $\mathrm{D}$ & \\
\hline 2 & WT & 0.055 & 13 & $\mathrm{~L}$ & 13.53 & 24 & WT & 0.08 \\
\hline 3 & $\mathrm{D}$ & 0.71 & 14 & $\mathrm{D}$ & 0.96 & 25 & $\mathrm{~L}^{3}$ & 13.50 \\
\hline 4 & WT & 0.047 & 15 & WT & 0.12 & 26 & $\mathrm{~S}^{4}$ & $8.00^{\circ}$ \\
\hline 5 & $\mathrm{D}$ & 0.64 & 16 & $\mathrm{~L}$ & 11.75 & 27 & $\mathrm{D}$ & 0.59 \\
\hline 6 & WT & 0.036 & 17 & $\mathrm{D}$ & 0.74 & 28 & WT & 0.07 \\
\hline 7 & $\mathrm{D}$ & 0.55 & 18 & WT & 0.13 & 29 & $\mathrm{D}$ & 0.45 \\
\hline 8 & WT & 0.037 & 19 & $\mathrm{~L}$ & 13.91 & 30 & WT & 0.07 \\
\hline 9 & $\mathrm{D}$ & 0.54 & 20 & $\mathrm{D}$ & 0.59 & 31 & $\mathrm{~L}$ & 5.10 \\
\hline 10 & WT & 0.035 & 21 & WT & 0.12 & 32 & $\mathrm{D}$ & 1.80 \\
\hline 11 & $\mathrm{D}$ & 0.84 & 22 & $\mathrm{~L}$ & 9.80 & 33 & WT & 0.06 \\
\hline
\end{tabular}

${ }^{1}$ D: Diameter (outer); ${ }^{2}$ WT: Wall Thickness; ${ }^{3}$ L: Length; ${ }^{4}$ S: Slope; ${ }^{5}$ BDV: Best Discovered Value

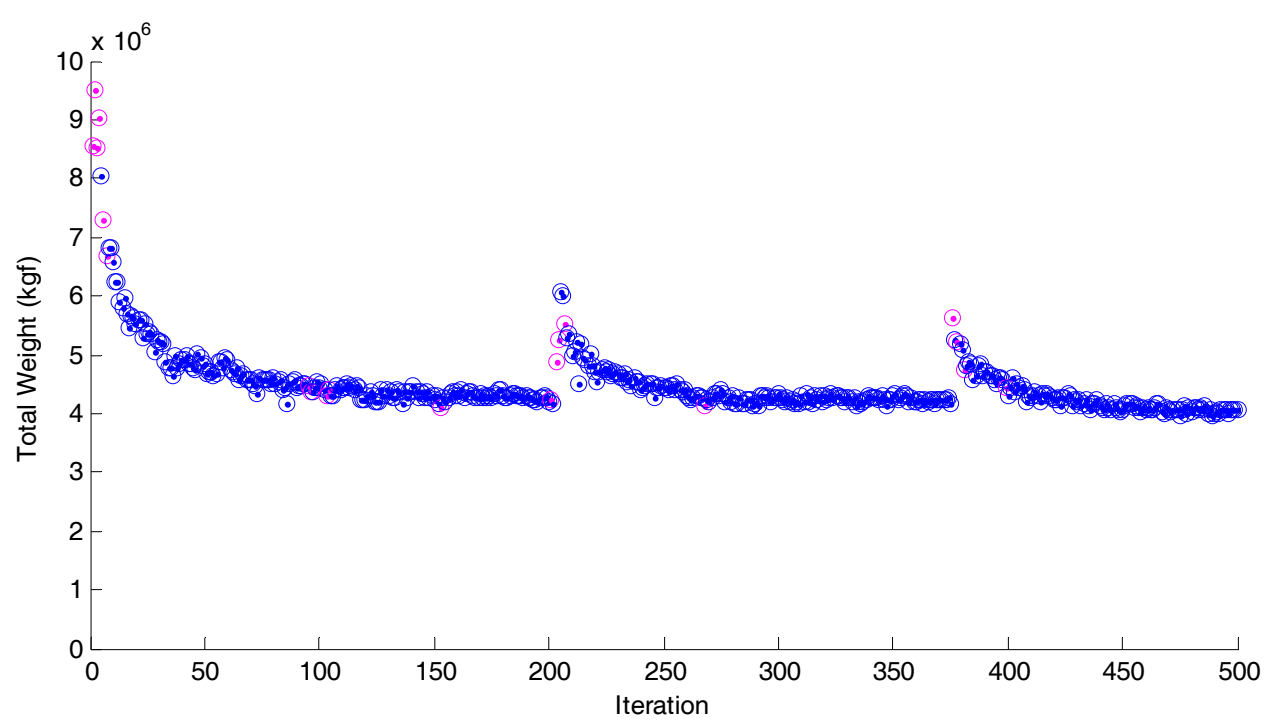

Figure 3: Path to convergence for the minimum weight of the quadropod structure.

\section{SUMMARY AND CONCLUSIONS}

A modified version of Genetic Algorithm with Domain Trimming (GADT) is presented in this study. The innovative technique is used to solve a least-weight topology and size optimization problem in the design of offshore wind turbine supporting structures. The GADT is used for solving the nonlinear optimization problem of 3D quatropod jacket offshore structure. In this optimum design formulation, the objective function is the material weight of the supporting truss; the design variables are the locations of the ends of the truss members as well as 
the cross-sectional areas of the truss members; the constraints are the stresses in members and the displacements of the joints. The constraints were handled using non-stationary (dynamically modified) penalty functions. The quatropod jacket problem is subjected to gravity, wind, wave and earthquake loading conditions. The results show that the (GADT) method is efficient in finding the best discovered optimal solution.

\section{REFERENCES}

[1] N. Haritos, "Introduction to the Analysis and Design of Offshore Structures - An Overview," Electronic Journal of Structural Engineering (EJSE), no. Special Issue: Loading on Structures, pp. 55-65, 2007.

[2] Q. Y. Duan, V. K. Gupta, and S. Sorooshian, "Shuffled complex evolution approach for effective and efficient global minimization," Journal of Optimization Theory and Applications, vol. 76, no. 3, pp. 501-521, Mar. 1993.

[3] N. D. Lagaros, M. Papadrakakis, and G. Kokossalakis, "Structural optimization using evolutionary algorithms," Computers \& Structures, vol. 80, no. 7-8, pp. 571-589, Mar. 2002.

[4] Q. S. Li, D. K. Liu, A. Y. T. Leung, N. Zhang, and Q. Z. Luo, "A multilevel genetic algorithm for the optimum design of structural control systems," International Journal for Numerical Methods in Engineering, vol. 55, no. 7, pp. 817-834, Nov. 2002.

[5] Q. S. Li, D. K. Liu, J. Tang, N. Zhang, and C. M. Tam, "Combinatorial optimal design of number and positions of actuators in actively controlled structures using genetic algorithms," Journal of Sound and Vibration, vol. 270, no. 4-5, pp. 611-624, Mar. 2004.

[6] R. J. Balling, R. R. Briggs, and K. Gillman, "Multiple Optimum Size/Shape/Topology Designs for Skeletal Structures Using a Genetic Algorithm," Journal of Structural Engineering, vol. 132, no. 7, pp. 1158-1165, Jul. 2006.

[7] R. E. Perez and K. Behdinan, "Particle swarm approach for structural design optimization," Computers \& Structures, vol. 85, no. 19-20, pp. 1579-1588, Oct. 2007.

[8] S. A. Barakat and S. Altoubat, "Application of evolutionary global optimization techniques in the design of RC water tanks," Engineering Structures, vol. 31, no. 2, pp. 332-344, Feb. 2009.

[9] G.-C. Luh and C.-Y. Lin, "Structural topology optimization using ant colony optimization algorithm," Applied Soft Computing, vol. 9, no. 4, pp. 1343-1353, Sep. 2009.

[10] S. Barakat and H. Ibrahim, "Application of shuffled complex evolution global optimization technique in the design of truss structures," in 2011 Fourth International Conference on Modeling, Simulation and Applied Optimization, 2011, pp. 1-6. 
[11] B. Zhang, Y. Wu, J. Lu, and K.-L. Du, "Evolutionary Computation and Its Applications in Neural and Fuzzy Systems," Applied Computational Intelligence and Soft Computing, vol. 2011, pp. 1-20, 2011.

[12] M. Kahraman and F. Erbatur, "A GA approach for simultaneous structural optimization," in Structural engineering mechanics and computation International conference, Structural engineering mechanics and computation, 2001, pp. 1147-1154.

[13] H. A. Eschenauer and N. Olhoff, "Topology optimization of continuum structures: A review," Applied Mechanics Reviews, vol. 54, no. 4, p. 331, Jul. 2001.

[14] M. P. Bendsoe and O. Sigmund, Topology Optimization: Theory, Methods, and Applications, 2nd ed. Berlin, Heidelberg: Springer Berlin Heidelberg, 2003.

[15] S. Joncas, M. J. de Ruiter, and F. van Keulen, "Preliminary Design of Large Wind Turbine Blades Using Layout Optimization Techniques," in 10th AIAA/ISSMO multidisciplinary analysis and optimization conference, 2004.

[16] M. Jureczko, M. Pawlak, and A. Mężyk, "Optimisation of wind turbine blades," Journal of Materials Processing Technology, vol. 167, no. 2-3, pp. 463-471, Aug. 2005.

[17] R. Zakhama, M. M. Abdalla, Z. Gürdal, and H. Smaoui, "Wind load effect in topology optimization problems," Journal of Physics: Conference Series, vol. 75, no. 1, p. 012048, Jul. 2007.

[18] P. Brøndsted, J. W. Holmes, and B. . Sørensen, "Wind rotor blades materials technology," European Sustainable Energy Review, vol. 2008, no. 2, pp. 36-41, 2008.

[19] S. Joncas, "Thermoplastic composite wind turbine blades; an integrated design approach," Technische Universiteit Delft, 2010.

[20] L.-C. Forcier and S. Joncas, "New Structural Design Concepts for Large Thermoplastic Wind Turbine Blades Using Structural Optimization Techniques," in 51st AIAA/ASME/ASCE/AHS/ASC Structures, Structural Dynamics, and Materials Conference, American Institute of Aeronautics and Astronautics, 2010.

[21] W. LIU and Y. Zhang, "Network Study of Plant Leaf Topological Pattern and Mechanical Property and its Application," Advances in Natural Science, vol. 3, no. 2, pp. 82-92, 2010.

[22] N. Buckney, A. Pirrera, S. D. Green, and P. M. Weaver, "Structural efficiency of a wind turbine blade,” Thin-Walled Structures, vol. 67, pp. 144-154, Jun. 2013.

[23] N. Buckney, S. Green, A. Pirrera, and P. M. Weaver, "On the structural topology of wind turbine blades," Wind Energy, vol. 16, no. 4, pp. 545-560, May 2013.

[24] "MATLAB Release 2013a." The MathWorks, Inc., Natick, Massachusetts, United States, 2013. 
[25] “SAP2000 ver. 16." Computers and Structures, Inc., Berkeley, California, USA, 2014.

[26] O. M. M. Nassif, "Optimization of Natural Rubber Seismic Isolation Systems with Supplemental Viscous Damping for Near-Field Ground Motion," College of Higher Studies and Research, University of Sharjah, 2014.

[27] American Institute of Steel Construction (AISC), "Specification for Structural Steel Buildings," ANSI/AISC 360-10, 2010. 